\title{
Análise da produção científica brasileira sobre etnobotânica: protocolo de scoping review
}

\author{
Analysis of brazilian scientific production on ethmobotanics: scoping review protocol \\ Análisis de la producción científica brasileña sobre etnobotánicos: protocolo de revisión de alcance
}

Recebido: 29/10/2021 | Revisado: 06/11/2021 | Aceito: 08/11/2021 | Publicado: 13/11/2021

\author{
Maria Andréa da Silva \\ ORCID: https://orcid.org/0000-0003-2121-3788 \\ Universidade Federal de Sergipe, Brasil \\ E-mail: andreafarma@yahoo.com.br \\ Fernando Henrique Oliveira de Almeida \\ ORCID: https://orcid.org/0000-0002-1728-9598 \\ Universidade Federal de Sergipe, Brasil \\ E-mail: fernandoalmeidafitofarm@gmail.com \\ Davi Cravo Teles dos Santos \\ ORCID: https://orcid.org/0000-0002-7812-3153 \\ Universidade Federal de Sergipe, Brasil \\ E-mail: davicravo10@gmail.com \\ Wellington Barros da Silva \\ ORCID: https://orcid.org/0000-0002-9691-6392 \\ Universidade Federal de Sergipe, Brasil \\ E-mail: wbarrosdasilva@gmail.com \\ Francilene Amaral da Silva \\ ORCID: https://orcid.org/0000-0001-6729-2843 \\ Universidade Federal de Sergipe, Brasil \\ E-mail: farmsilva@gmail.com
}

\begin{abstract}
Resumo
Este artigo apresenta o protocolo de revisão de escopo acerca da produção científica brasileira sobre etnobotânica. E tem como objetivo documentar os processos envolvidos no planejamento e na condução metodológica deste estudo inédito, examinando a extensão, o alcance e a natureza das atividades de pesquisa em etnobotânica no Brasil, com vistas a identificar as possíveis lacunas de pesquisa na literatura existente. Esta revisão será conduzida seguindo as diretrizes do Joanna Briggs Institute (JBI) e elaborada de acordo com os itens do PRISMA Extension for Scoping Reviews (PRISMA-ScR): Checklist and Explanation. Para tal, serão selecionados e revisados artigos científicos publicados nos idiomas: inglês, português e espanhol nas seguintes bases de dados: Web of Science, PubMed /Medline, Lilacs/BVS, Scopus e Scielo. Este protocolo foi registrado no Open Science Framework (OSF), sob doi: 10.17605/OSF.IO/N4XD3. Espera-se com esse estudo, mapear, demonstrar o cenário e o panorama dos estudos etnobotânicos realizados no Brasil, o que poderá orientar a realização de futuras pesquisas nessa temática em nosso país.
\end{abstract}

Palavras-chave: Comunidades tradicionais; Etnobotânica; Plantas medicinais; Uso tradicional.

\begin{abstract}
This article presents the scope review protocol on Brazilian scientific production on ethnobotany. And it aims to document the processes involved in the planning and methodological conduct of this unprecedented study, examining the extent, scope and nature of research activities in ethnobotany in Brazil with a view to identifying possible research gaps in the existing literature. This review will be conducted following the guidelines of the Joanna Briggs Institute (JBI) and prepared in accordance with the items of the PRISMA Extension for Scoping Reviews (PRISMA-ScR): Checklist and Explanation. To this end, scientific articles published in the following languages will be selected and reviewed: English, Portuguese and Spanish in the following databases: Web of Science, PubMed /Medline, Lilacs/BVS, Scopus, and Scielo. This protocol has been registered in the Open Science Framework (OSF), under doi: 10.17605/OSF.IO/N4XD3. It is hoped that this study will map and demonstrate the scenario and panorama of ethnobotanical studies carried out in Brazil, which may guide the conduct of future research on this topic in our country.
\end{abstract}

Keywords: Ethnobotany; Medicinal plants; Traditional communities; Traditional use. 


\begin{abstract}
Resumen
Este artículo presenta el protocolo de revisión del alcance de la producción científica brasileña en etnobotánica. Y tiene como objetivo documentar los procesos involucrados en la planificación y conducción metodológica de este estudio sin precedentes, examinando el alcance, alcance y naturaleza de las actividades de investigación en etnobotánica en Brasil con miras a identificar posibles lagunas de investigación en la literatura existente. Esta revisión se llevará a cabo siguiendo las pautas del Instituto Joanna Briggs (JBI) y se preparará de acuerdo con los ítems de la Extensión PRISMA para Revisiones de Alcance (PRISMA-ScR): Lista de Verificación y Explicación. Para ello, se seleccionarán y revisarán artículos científicos publicados en los siguientes idiomas: inglés, portugués y español en las siguientes bases de datos: Web of Science, PubMed / Medline, Lilacs / BVS, Scopus y Scielo. Este protocolo ha sido registrado en Open Science Framework (OSF), bajo doi: 10.17605/OSF.IO/N4XD3. Se espera que este estudio mapee y demuestre el escenario y panorama de los estudios etnobotánicos realizados en Brasil, que puedan orientar la conducción de futuras investigaciones sobre este tema en nuestro país.
\end{abstract}

Palabras clave: Comunidades tradicionales; Etnobotánica; Plantas medicinales; Uso tradicional.

\title{
1. Introdução
}

O Brasil além de possuir a maior biodiversidade do planeta, detém também uma rica diversidade étnica e cultural, o que nos propicia um valioso conhecimento tradicional ligado ao uso de plantas medicinais (Brasil, 2016). O saber tradicional, que se perpetua durante séculos e é transmitido de uma geração à outra, tem estimulado estudos sobre o uso de plantas medicinais em diversas comunidades levando ao conhecimento das propriedades terapêuticas das plantas e inclusive à confirmação científica do seu uso (Maciel et al., 2002).

A interação dos homens com os recursos da natureza remonta os primórdios da humanidade. Cientificamente, esta interação é estudada pela etnobotânica que investiga as relações entre as plantas e o ser humano, numa perspectiva antropológica-cultural, visto que analisa a interação humana com as plantas, de forma associada com seus costumes, ritos, crenças, dentre outros aspectos. Dentro deste contexto, insere-se a etnofarmacologia, que estuda o conhecimento popular relacionado aos sistemas tradicionais de medicina, resgatando informações junto à população que faz uso da flora medicinal (Elisabetsky, 2003). Ambas estão fortemente ligadas e têm sido muito usadas na busca por substâncias de ação terapêutica (Monteiro \& Brandelli, 2017).

Segundo Monteiro e Brandelli (2017, p.18), a etnobotânica e a etnofarmacologia são ramos da ciência denominada Etnobiologia, que abrange o estudo das interações dos grupos humanos com o ambiente, ou seja, suas percepções, conceitos e classificações. A etnobotânica é um meio de pesquisa que favorece a relação da espécie humana com a grande diversidade vegetal e contribui de forma significativa no tratamento das enfermidades humanas e nos rituais religiosos (Franco et al., 2011).

A descoberta de substâncias biologicamente ativas que podem levar a cura de doenças foi, em sua maior parte, originada a partir de estudos etnofarmacológicos (Albuquerque \& Hanazaki, 2006; Maciel et al., 2002; Süntar, 2020). A importância de estudos multidisciplinares envolvendo a etnobotânica, a química e a farmacologia enriquecem os conhecimentos sobre produtos de origem vegetal (Maciel et al., 2002). O uso dos recursos naturais está ligado às comunidades locais, como por exemplo, em pequenas propriedades agrícolas, comunidades caiçaras, quilombolas e indígenas, que fazem o uso de plantas para fins ornamentais e como medicinais. As plantas medicinais são geralmente cultivadas em hortas, quintais ou coletadas em mata, e utilizadas de acordo com o conhecimento acumulado e transmitido de antepassados (Sales et al., 2015).

De acordo com dados da Web of Science, o Brasil, é o segundo país que mais publica artigos científicos na área de etnobotânica, ficando atrás apenas, dos Estados Unidos da América (EUA). Grande parte dessa produção científica advém da nossa grande biodiversidade, fonte do saber tradicional de diversas comunidades em nosso país.

Diante deste contexto, torna-se de grande importância a realização de estudos que nos tragam um panorama do que tem sido produzido no Brasil em torno dessa temática, tanto para engrandecer nossos conhecimentos sobre tais pesquisas como 
também para nos mostrar de que forma elas estão sendo realizadas. Neste sentido, faz-se necessária uma avaliação mais aprofundada e abrangente, que nos traga uma síntese de pontos específicos destes estudos (Albuquerque \& Hanazaki, 2006; Maciel et al., 2002). Para isso, o melhor modelo de estudo disponível na literatura é a revisão de escopo (Grant \& Booth, 2009).

Os estudos do tipo revisão de escopo buscam mapear o conhecimento sobre determinada área e têm sido bastante utilizados nos últimos anos podendo ser usadas de várias maneiras, como, por exemplo, para identificação de lacunas de pesquisa e síntese de descobertas científicas. As revisões de escopo também podem ser usadas para explorar a extensão da literatura sobre um determinado tema, ajudar a identificar os parâmetros adequados de uma revisão (ou seja, definir a população-alvo, intervenção, comparação, resultados) e identificar a necessidade de realização de uma revisão sistemática. Normalmente, as revisões de escopo não avaliam a qualidade dos estudos incluídos o que pode limitar a síntese dos dados e a sua interpretação (Armstrong et al., 2011; Cordeiro \& Soares, 2019; Tricco et al., 2016).

Seguindo às recomendações propostas pelas diretrizes metodológicas do Instituto Joanna Briggs (JBI), a elaboração do protocolo deve anteceder à realização de uma revisão de escopo, consistindo desta forma, na primeira etapa antes da realização da revisão em si, com o intuito de dar transparência e demonstrar a estrutura das etapas que conduzirá a elaboração da revisão, para com isso reduzir possíveis distorções no método de recuperação e escolha dos artigos, consolidando e uniformizando o processo de seleção e leitura entre os revisores, e possibilitando rigor metodológico e reprodutibilidade nas diversas fases do estudo (Tricco et al., 2018).

Sendo assim, o estudo a que se refere este protocolo, pretende responder à seguinte pergunta: "O que tem sido produzido no Brasil sobre etnobotânica?". Deste modo, buscando responder a essa indagação, o objetivo deste estudo será realizar uma revisão de escopo para analisar a produção científica brasileira sobre etnobotânica. Para tal, serão analisadas a extensão, o alcance e a natureza das atividades de pesquisa em etnobotânica no Brasil, bem como, serão identificadas as possíveis lacunas na literatura existente. $O$ fato de não existir ainda uma revisão de escopo nessa temática, também nos confirma a importância de sua realização.

\section{Metodologia}

O presente estudo refere-se à construção do protocolo da revisão de escopo: "Análise da produção científica brasileira sobre etnobotânica: uma revisão de escopo" e seguirá às recomendações exigidas pela diretriz Preferred Reporting Items for Systematic reviews and Meta-Analyses extension for Scoping Reviews (PRISMA-ScR) que consiste em um roteiro para guiar a redação do relatório de revisão de escopo, e demonstra as seções obrigatórias na estruturação da pesquisa que são Título, Resumo Estruturado, Introdução, Método, Resultados, Discussão e Financiamento (Tricco et al., 2018). O protocolo está registrado no Open Science Framework (doi:10.17605/OSF.IO/N4XD3) e a revisão proposta será conduzida de acordo com a metodologia do JBI para revisões de escopo (Peters et al., 2020).

\subsection{Elaboração da pergunta de pesquisa}

As questões de pesquisa do estudo de escopo são de natureza ampla, pois o foco é resumir a amplitude das evidências. Sendo recomendado que os pesquisadores combinem a uma ampla questão de pesquisa um escopo de investigação claramente articulado. Este inclui a definição do conceito, população-alvo e resultados de interesse para esclarecer o foco do estudo e estabelecer uma estratégia de pesquisa eficaz (Arksey \& O’Malley, 2005; Colquhoun et al., 2014; Daudt et al., 2013; Levac et al., 2010). 
O JBI Manual for Evidence Synthesis (2020) propõe que a estratégia "PCC" seja usada para construir a pergunta de pesquisa em revisões de escopo (Peters et al., 2020). O mnemônico "PCC" significa População, Conceito e Contexto. Desse modo foram definidos para o presente estudo:

-População (P): Comunidades tradicionais (quilombolas, afro-brasileiros, ribeirinhos, jangadeiros, indígenas, rezadeiras e outras) ou grupos populacionais que utilizam as plantas medicinais em determinados contextos de uso;

\section{-Conceito (C): Etnobotânica; e \\ -Contexto (C): Plantas medicinais no âmbito dos estudos produzidos no Brasil.}

A partir de tais componentes elaborou-se a seguinte pergunta norteadora da revisão: "O que tem sido produzido no

\section{Brasil sobre etnobotânica?"}

Com base nos objetivos específicos foram formuladas quatro perguntas secundárias, que são:

1. Como ocorre o mapeamento da produção científica publicada no Brasil sobre etnobotânica?

2. Quais as espécies de plantas são utilizadas terapeuticamente pelas comunidades citadas nos estudos?

3. Quais os diferentes desenhos dos estudos recuperados na busca?

4. Quais são as potencialidades e as limitações dos estudos etnobotânicos apontados na literatura?

\subsection{Estratégia de busca e Identificação dos estudos}

Para a identificação dos estudos relevantes, serão consultadas as bases de dados Web of Science, PubMed /Medline, Lilacs/BVS, Scopus e Scielo, e serão selecionados artigos nos idiomas português, inglês e espanhol. Não selecionaremos nenhum lapso temporal, de forma que todos os estudos que atendam aos critérios de elegibilidade e constem nessas bases de dados possam ser utilizados.

Após a elaboração da pergunta de pesquisa seguimos na elaboração da estratégia de busca (Quadros 1-4), seguindo as orientações de Araújo (2020), com base nos objetivos, problema e pergunta de pesquisa.

Quadro 1: Elaboração da Estratégia de Busca - População

\begin{tabular}{|l|l|}
\hline & \multicolumn{1}{|c|}{ POPULAÇÃO } \\
\hline Extração & Comunidades Tradicionais \\
\hline Conversão & Communities \\
\hline $\begin{array}{l}\text { Comblês } \\
\text { Inação/ }\end{array}$ & $\begin{array}{l}\text { community; communities; traditional use; traditional use; traditional knowledge; } \\
\text { traditional population; traditional populations; traditional practice; traditional } \\
\text { practices; traditional belief; traditional beliefs; traditional people; popular use; } \\
\text { popular uses; }\end{array}$ \\
\hline $\begin{array}{l}\text { Combinação/ } \\
\text { Português }\end{array}$ & $\begin{array}{l}\text { comunidade; comunidades; uso tradicional; usos tradicionais; conhecimento } \\
\text { tradicional; conhecimentos tradicionais; população tradicional; populações } \\
\text { tradicionais; saber tradicional; saberes tradicionais; prática tradicional; práticas } \\
\text { tradicionais; crença tradicional; crenças tradicionais; povo tradicional; povos } \\
\text { tradicionais; costume tradicional; costumes tradicionais; uso popular; usos populares; } \\
\text { raizeira; raizeiro; raizeiras; raizeiros; quilombola; quilombolas; indígena; indígenas; } \\
\text { rezadeiras; rezadeiros; }\end{array}$ \\
\hline $\begin{array}{l}\text { Combinação/ } \\
\text { Espanhol }\end{array}$ & $\begin{array}{l}\text { comunidad; comunidades; } \text { uso tradicional; } \text { usos tradicionales; conocimiento } \\
\text { tradicional; poblacion tradicional; poblaciones tradicionales; práctica tradicional; } \\
\text { prácticas tradicionales; creencia tradicional; } \text { creencias tradicionales; pueblo } \\
\text { tradicional; pueblos tradicionales; costumbre tradicional; costumbres tradicionales; } \\
\text { curandeiro; }\end{array}$ \\
\hline
\end{tabular}

Fonte: Adaptado de Araújo (2020). 
Quadro 2: Elaboração da Estratégia de Busca - Conceito.

\begin{tabular}{|l|c|}
\hline & CONCEITO \\
\hline Extração & Etnobotânica \\
\hline Conversão & Ethnobotany \\
\hline Combinação/ Inglês & Ethnobotany \\
\hline Combinação/ Português & Etnobotânica \\
\hline Combinação/ Espanhol & \\
& Etnobotánica \\
\hline
\end{tabular}

Fonte: Adaptado de Araújo (2020).

Quadro 3: Elaboração da Estratégia de Busca - Contexto.

\begin{tabular}{|l|l|}
\hline & \multicolumn{1}{|c|}{ CONTEXTO } \\
\hline Extração & Plantas Medicinais no Brasil \\
\hline Conversão & Medicinal Plants; Brazil \\
\hline Combinação/ & $\begin{array}{l}\text { medicinal plant; medicinal plants; medicinal herb; medicinal herbs; pharmaceutical herb; } \\
\text { pharmaceutical herbs; healing herb; healing herbs; healing plant; healing plants; }\end{array}$ \\
\hline Comglês & $\begin{array}{l}\text { planta medicinal; plantas medicinais; erva medicinal; ervas medicinais; erva farmacêutica; ervas } \\
\text { farmacêuticas; erva curativa; ervas curativas; planta curativa; plantas curativas; }\end{array}$ \\
\hline Combinação/ & $\begin{array}{l}\text { planta medicinal; plantas medicinales; hierba medicinal; hierbas medicinales; hierba } \\
\text { farmacêutica; hierbas farmacêuticas; hierba curativa; } \text { hierba curativas; }\end{array}$ \\
\hline
\end{tabular}

Fonte: Adaptado de Araújo (2020). 
Quadro 4: Elaboração da Estratégia de Busca - Construção/ Uso

\begin{tabular}{|c|c|}
\hline Construção/Uso & $\begin{array}{l}\text { ("community" OR "communities" OR "traditional use" OR "traditional uses" OR } \\
\text { "traditional knowledge" OR "traditional population" OR "traditional populations" OR } \\
\text { "traditional practice" OR "traditional practices" OR "traditional belief" OR "traditional } \\
\text { beliefs" OR "traditional people" OR "popular use" OR "popular uses" OR } \\
\text { "comunidade" OR "comunidades" OR "uso tradicional" OR "usos tradicionais" OR } \\
\text { "conhecimento tradicional" OR "conhecimentos tradicionais" OR "população } \\
\text { tradicional" OR "populações tradicionais" OR "saber tradicional" OR "saberes } \\
\text { tradicionais" OR "prática tradicional" OR "práticas tradicionais" OR "crença } \\
\text { tradicional" OR "crenças tradicionais" OR "povo tradicional" OR "povos tradicionais" } \\
\text { OR "costume tradicional" OR "costumes tradicionais" OR "uso popular" OR "usos } \\
\text { populares" OR "raizeira" OR "raizeiro" OR "raizeiras" OR "raizeiros" OR } \\
\text { "quilombola" OR "quilombolas" OR "indígena" OR "indígenas" OR "rezadeiras" OR } \\
\text { "rezadeiros" OR "comunidad" OR "comunidades" OR "uso tradicional" OR "usos } \\
\text { tradicionales" OR "conocimiento tradicional" OR "poblacion tradicional" OR } \\
\text { "poblaciones tradicionales" OR "práctica tradicional" OR "prácticas tradicionales" OR } \\
\text { "creencia tradicional" OR "creencias tradicionales" OR "pueblo tradicional" OR } \\
\text { "pueblos tradicionales" OR "costumbre tradicional" OR "costumbres tradicionales" OR } \\
\text { "curandero") AND ("ethnobotany" OR "etnobotânica" OR "etnobotánica") AND } \\
\text { ("medicinal plant" OR "medicinal plants" OR "medicinal herb" OR "medicinal herbs" } \\
\text { OR "pharmaceutical herb" OR "pharmaceutical herbs" OR "healing herb" OR "healing } \\
\text { herbs" OR "healing plant" OR "healing plants" OR "planta medicinal" OR "plantas } \\
\text { medicinais" OR "erva medicinal" OR "ervas medicinais" OR "erva farmacêutica" OR } \\
\text { "ervas farmacêuticas" OR "erva curativa" OR "ervas curativas" OR "planta curativa" } \\
\text { OR "plantas curativas" OR "planta medicinal" OR "plantas medicinales" OR "hierba } \\
\text { medicinal" OR "hierbas medicinales" OR "hierba farmacéutica" OR "hierbas } \\
\text { farmacéuticas" OR "hierba curativa" OR "hierba curativas") }\end{array}$ \\
\hline
\end{tabular}

Fonte: Adaptado de Araújo (2020).

Para os itens "conceito" e "contexto" da estratégia "PCC" foram selecionados descritores disponíveis nos Descritores de Ciências da Saúde (DeCS) e no Medical Subject Headings (MeSH) nas bases PubMed /Medline e Lilacs/BVS sempre associando os mesmos termos como busca livre, de acordo com as particularidades de cada base de dados consultada, como demonstrado no Quadro 5. Já para as bases Web of Science, Scopus e Scielo a busca foi realizada apenas com os termos como busca livre. Para o item "população" da estratégia "PCC" os termos foram utilizados na estratégia como busca livre, visto que os mesmos não estavam classificados como DeCS ou MeSH.

Quadro 5: Descritores Utilizados (DESC/MESH)

\begin{tabular}{|l|l|l|}
\hline Bases & PubMed /Medline & Lilacs/BVS \\
\hline Descritores-Conceito & "ethnobotany" (MESH) & $\begin{array}{l}\text { "ethnobotany" OR "etnobotânica" OR } \\
\text { "etnobotánica" (DESC/MESH) }\end{array}$ \\
\hline Descritores-Contexto & "Plants, Medicinal" (MESH) & $\begin{array}{l}\text { "Plants, Medicinal" OR "Plantas } \\
\text { Medicinais" OR "Plantas Medicinales" } \\
\text { (DESC/MESH) }\end{array}$ \\
\hline
\end{tabular}

Fonte: Autores (2021). 
A estratégia de busca foi construída por meio da combinação dos DeCS, dos MeSH terms e dos termos de busca livre (Quadro 1, 2, 3, 4 e 5). Utilizando como base o acrônimo PCC, a aplicação dos operadores booleanos: AND/E, OR/OU e o cruzamento dos descritores e termos de busca livres, considerando os idiomas português, inglês e espanhol e as particularidades de cada base de dados pesquisada.

Como o objetivo é analisar os estudos produzidos no Brasil, foi utilizado o filtro "país" nas bases de dados Web of Science, Lilacs/BVS, Scopus e Scielo, e na base PubMed /Medline, que não dispõe deste filtro, incluímos na estratégia de busca o termo "Brasil" com as suas variações ("Brazil" OR "Brazilian" OR "Brasil" OR "brasileiro" OR "brasileira" OR "brasileno" OR "brasilena") e também utilizamos o MeSH terms "Brazil" disponibilizado na referida base.

\subsection{Critérios de Elegibilidade}

\subsubsection{Critérios de inclusão}

1. Serão incluídos estudos primários publicados em português, inglês ou espanhol que tenham sido produzidos no Brasil.

2. Serão considerados estudos etnobotânicos, quantitativos, qualitativos ou de métodos mistos, a fim de poder abranger diferentes aspectos (também poderão ser incluídos estudos que tragam aspectos da etnobotânica em estudos de etnofarmacologia ou etnomedicina). Desde que tenham tido a participação de algum representante de comunidades tradicionais de diferentes povos ou etnias (por exemplo: raizeiras, raizeiros, quilombolas, afro-brasileiros, afro-brasileiras, ribeirinhas, ribeirinhos, jangadeiras, jangadeiros, indígenas, rezadeiras).

3. Ou ainda, estudos que tenham a participação, segundo relata Albuquerque e Hanazaki (2006, p. 679):

[...] de pessoas que participem de determinados grupos populacionais que utilizam as plantas medicinais em determinados contextos de uso, enfatizando a busca pelo conhecimento construído localmente a respeito de seus recursos naturais e a aplicação que fazem deles em seus sistemas de saúde e doença (Albuquerque \& Hanazaki, 2006, p. 679).

E que de alguma forma, essas pessoas consultadas, tenham contribuído com a transmissão de seus saberes sobre plantas medicinais durante a realização do estudo. Objetivando também a manutenção do registro e a preservação do conhecimento botânico tradicional das comunidades estudadas.

Caso algum estudo esteja indisponível na íntegra nas bases de dados utilizadas, serão realizadas tentativas de obtenção dos mesmos, seja através do envio de e-mails para os autores como também através da consulta em plataformas como, por exemplo: Comut ou ResearchGate.

\subsubsection{Critérios de Exclusão}

Serão excluídos os estudos do tipo revisão de literatura como, por exemplo, as revisões narrativas, revisões integrativas, revisões bibliométricas, revisões sistemáticas, overview, dentre outras. Como também serão excluídos estudos cuja obtenção na íntegra não tenha sido possível.

\subsection{Seleção dos estudos}

Os estudos selecionados nas bases de dados serão incluídos automaticamente no Rayyan ${ }^{\circledR}$ QRCI (Qatar Computing Research Institute), uma ferramenta virtual que auxilia na triagem de duplicidades e análise de títulos e resumos em estudos de revisão e analisados por três revisores independentes.

A seleção dos artigos ocorrerá em duas fases: 
- $\quad$ Na primeira etapa, realizada no Rayyan $^{\circledR}$, os estudos duplicados serão removidos e os demais serão submetidos à leitura do título e do resumo por dois revisores independentes, considerando a inclusão de estudos que respondam aos objetivos da pesquisa. Se existir (em) discordância(s) entre os revisores nessa fase, a(s) mesma(s) será (ão) resolvida(s) por um terceiro revisor.

- $\quad$ Na segunda fase será realizada a leitura na íntegra dos artigos selecionados na primeira fase, por dois revisores independentes e suas discordâncias serão resolvidas pelo terceiro revisor da equipe. Aqui os revisores farão uma análise dos artigos para suprir possíveis dúvidas ou esclarecer informações que não tenham ficado claras no momento da leitura dos títulos e resumos e terão a responsabilidade de verificar se o artigo atende aos critérios de elegibilidade e se os mesmos respondem ao objetivo da revisão. Todas as exclusões realizadas nesta fase serão justificadas. As referências dos artigos também serão avaliadas, considerando a possibilidade de incluir estudos que não foram recuperados no momento da busca nas bases de dados selecionadas. Esta etapa também contemplará a extração e sumarização das informações dos artigos incluídos que respondam ao objetivo da revisão. Todos os estudos incluídos nessa fase serão divididos entre os três revisores, que irão preencher a matriz de síntese da extração de dados.

\subsection{Extração dos dados}

Para a análise e posterior síntese dos artigos serão utilizadas três tabelas de extração dos dados para uma melhor compreensão e visualização dos resultados: Tabelas 1,2 e 3, que serão preenchidas de forma independente pelos dois revisores. A Tabela 1 trará informações gerais sobre os estudos, também abrangendo dados bibliográficos básicos; a Tabela 2 trará informações referentes ao "conceito" e "população" que compõem a estratégia PCC e a tabela 3 trará informações sobre o elemento "contexto". Isso permitirá descrever de forma mais clara os principais aspectos relacionados à realização de estudos etnobotânicos no Brasil. As informações coletadas nessa fase serão armazenadas em um banco de dados eletrônico criado no programa Microsoft Excel for Windows ${ }^{\circledR}$.

Possíveis questionamentos e dúvidas sobre as extrações serão dirimidas em consenso com os dois revisores principais e, quando não resolvidos, serão tratados com o terceiro revisor da equipe.

Os formulários de extração inicialmente preparados para essa revisão, serão testados e validados previamente de maneira independente por dois revisores da equipe. No decorrer desse processo, poderão ocorrer ajustes e/ou alterações nas informações que serão extraídas dos artigos científicos, decididas após discussões e consultas aos membros da equipe, com o objetivo de garantir a consistência e a clareza na extração das informações. 
Tabela 1: Informações Gerais.

\begin{tabular}{|c|c|c|c|c|c|c|c|c|}
\hline Autores, Ano & $\begin{array}{l}\text { Título do } \\
\text { Periódico }\end{array}$ & Qualis & Instituição & Local & Objetivo & Potencialidades & Limitações & $\begin{array}{l}\text { Principais } \\
\text { Resultados }\end{array}$ \\
\hline
\end{tabular}

Tabela 2: Informações Específicas- CONCEITO e POPULAÇÃO.

\begin{tabular}{llccccc}
\hline Autores, Ano & Tipo de & Comunidades & Período de & Duração & Métodos & Formas de \\
Estudo & alvo & realização & Utilizados & retorno às \\
& & &
\end{tabular}

Fonte: Autores (2021).

Tabela 3: Informações Específicas- CONTEXTO.

\begin{tabular}{llcccc}
\hline Autores, Ano & Plantas & Usos & Local de \\
Citadas & $\begin{array}{c}\text { Terapêuticos } \\
\text { Atribuídos }\end{array}$ & $\begin{array}{c}\text { Identificação } \\
\text { do Material } \\
\text { Vegetal }\end{array}$ & $\begin{array}{c}\text { Tratamento } \\
\text { do material } \\
\text { vegetal }\end{array}$ & $\begin{array}{c}\text { Tipo de } \\
\text { Intervenção }\end{array}$ \\
\hline
\end{tabular}

Fonte: Autores (2021).

\subsection{Sumarização dos resultados}

Após a etapa de extração, as informações coletadas serão organizadas, analisadas e interpretadas de forma a proporcionar a elaboração de uma descrição dos resultados de acordo com o objetivo do estudo e das questões norteadoras da revisão.

Já os resultados da revisão de escopo em si, serão apresentados e demonstrados por meio de tabelas, fluxogramas e/ou outros meios que facilitem a visualização e também serão sintetizados e apresentados em formato narrativo, possibilitando 
assim uma melhor descrição e caracterização dos dados com o objetivo de agrupar os conhecimentos pertinentes sobre o tema proposto,

\section{Resultados esperados}

A organização e o mapeamento das informações presentes nos artigos científicos que serão incluídos no estudo trarão dados importantes sobre o cenário e o panorama dos estudos etnobotânicos realizados no Brasil, o que poderá orientar a realização de futuras pesquisas nessa temática em nosso país, tão rico em biodiversidade, saberes tradicionais e produção de conhecimento científico.

\section{Considerações Finais}

Desta forma esse protocolo visa explicar e sistematizar as etapas metodológicas para a realização de uma revisão de escopo sobre o referido tema, objetivando reduzir possíveis distorções nesse processo, trazendo transparência e conformidade entre os revisores da equipe no decorrer do estudo.

\section{Declaração de Conflito de Interesse}

Os autores declaram não possuir conflito de interesse.

\section{Financiamento}

Este estudo está sendo financiado pelo CNPQ - Conselho Nacional de Desenvolvimento Científico e Tecnológico, através de bolsa de estudo, concedida à M.A.S.

\section{Referências}

Albuquerque, U. P. de., \& Hanazaki, N. (2006). As pesquisas etnodirigidas na descoberta de novos fármacos de interesse médico e farmacêutico: fragilidades e pespectivas. Revista Brasileira de Farmacognosia, 16, 678-689. https://doi.org/10.1590/s0102-695x2006000500015

Araújo, W. C. O. (2020). Recuperação da informação em saúde: construção, modelos e estratégias. In Convergências em Ciência da Informação (Vol. 3, Issue 2). https://doi.org/10.33467/conci.v3i2.13447

Arksey, H., \& O’Malley, L. (2005). Scoping studies: Towards a methodological framework. International Journal of Social Research Methodology, 8(1), 1932. https://doi.org/10.1080/1364557032000119616

Armstrong, R., Hall, B. J., Doyle, J., \& Waters, E. (2011). "Scoping the scope" of a cochrane review. Journal of Public Health, 33(1), 147-150. https://doi.org/10.1093/pubmed/fdr015

Brasil. (2016). Ministério da Saúde. Secretaria de Ciência, Tecnologia e Insumos Estratégicos. Departamento de Assistência Farmacêutica. Política e Programa Nacional de Plantas Medicinais e Fitoterápicos. Brasília, DF: Ministério Da Saúde. http://bvsms.saude.gov.br/ bvs/publicacoes/politica_programa_nacional_plantas_medicinais_fitoterapicos.pdf.

Colquhoun, H. L., Levac, D., O’Brien, K. K., Straus, S., Tricco, A. C., Perrier, L., Kastner, M., \& Moher, D. (2014). Scoping reviews: Time for clarity in definition, methods, and reporting. Journal of Clinical Epidemiology, 67(12), 1291-1294. https://doi.org/10.1016/j.jclinepi.2014.03.013

Cordeiro, L., \& Soares, C. B. (2019). Revisão de escopo: potencialidades para a síntese de metodologias utilizadas em pesquisa primária qualitativa. Bis, 3743.

Daudt, H. M. L., Van Mossel, C., \& Scott, S. J. (2013). Enhancing the scoping study methodology: A large, inter-professional team's experience with Arksey and O'Malley's framework. BMC Medical Research Methodology, 13(1), 1-9. https://doi.org/10.1186/1471-2288-13-48

Elisabetsky, E. (2003). Etnofarmacologia. Ciência e Cultura, 55(3), 35-36.

Franco, F., Lamano-Ferreira, A. P. do N., \& Ferreira, M. L. (2011). Etnobotânica: Aspectos Históricos E Aplicativos Desta Ciência. Caderno de Cultura e Ciência, 10(2), 17-23. https://doi.org/10.14295/cad.cult.cienc.v10i2.407

Grant, M. J., \& Booth, A. (2009). A typology of reviews: An analysis of 14 review types and associated methodologies. Health Information and Libraries Journal, 26(2), 91-108. https://doi.org/10.1111/j.1471-1842.2009.00848.x 
Research, Society and Development, v. 10, n. 14, e545101422493, 2021

(CC BY 4.0) | ISSN 2525-3409 | DOI: http://dx.doi.org/10.33448/rsd-v10i14.22493

Levac, D., Colquhoun, H., \& O’Brien, K. K. (2010). Scoping studies: Advancing the methodology. Implementation Science, 5(69), 1-9. https://doi.org/10.1186/1748-5908-5-69

Maciel, M. A. M., Pinto, A. C., Veiga Jr, V. F., Grynberg, N. F., \& Echevarria, A. (2002). Plantas Medicinais: A Necessidade De Estudos Multidisciplinares. Química Nova, 25(3), 429-438. https://doi.org/10.1590/S0100-40422002000300016

Peters, M., Godfrey, C., McInerney, P., Munn, Z., Trico, A., \& Khalil, H. (2020). Scoping Reviews. In Aromataris E \& Munn Z (Eds.), JBI Manual for Evidence Synthesis. JBI. https://doi.org/10.46658/JBIMES-20-12

Sales, M., Sartor, E., \& Gentilli, R. (2015). Etnobotânica e etnofarmacologia : medicina tradicional e bioprospecção de fitoterápicos. Revista Salus Journal of Health Sciences, 1(1), 17-26. https://doi.org/10.5935/2447-7826.20150003

Monteiro, S. da C., \& Brandelli, C. L. C. (2017). Etnobotânica. In Simone de Fraga (Ed.), Farmacobotânica: Aspectos Teóricos e Aplicação (1ª , pp. 15-25). Artmed. https://www.amazon.com.br/Farmacobotânica-aplicação-Siomara-Cruz-Monteiro/dp/8582714408

Süntar, I. (2020). Importance of ethnopharmacological studies in drug discovery: role of medicinal plants. Phytochemistry Reviews, 19(5), 1199-1209. https://doi.org/10.1007/s11101-019-09629-9

Tricco, A. C., Lillie, E., Zarin, W., O’Brien, K., Colquhoun, H., Kastner, M., Levac, D., Ng, C., Sharpe, J. P., Wilson, K., Kenny, M., Warren, R., Wilson, C., Stelfox, H. T., \& Straus, S. E. (2016). A scoping review on the conduct and reporting of scoping reviews. BMC Medical Research Methodology, 16(1), 1-10. https://doi.org/10.1186/s12874-016-0116-4

Tricco, A. C., Lillie, E., Zarin, W., O’Brien, K. K., Colquhoun, H., Levac, D., Moher, D., Peters, M. D. J., Horsley, T., Weeks, L., Hempel, S., Akl, E. A., Chang, C., McGowan, J., Stewart, L., Hartling, L., Aldcroft, A., Wilson, M. G., Garritty, C., \& Straus, S. E. (2018). PRISMA extension for scoping reviews (PRISMA-ScR): Checklist and explanation. Annals of Internal Medicine Research and Reporting Methods, 169(7), 467-473. https://doi.org/10.7326/M180850

Web of Science. (2021). Resultados de coleção principal da Web of Science para "Ethnobotany". https://www-webofscience.ez20.periodicos.capes.gov.br/ wos/woscc/summary/d697d275-61b1-4df4-b0f6-3ec84e00141c-007361ac/relevance/1 